\title{
Business process assessment using discrete simulation
}

\author{
I. P. Tatsiopoulos ${ }^{\text {I) }}$, N. A. Panayiotou ${ }^{\text {(\&2) }}$, S. T. Ponis ${ }^{\text {(\&3) }}$, \\ ${ }^{1)}$ National Technical University of Athens \\ ${ }^{2)} M B A$, Lancaster University, UK \\ ${ }^{3)}$ Mechanical Engineer, NTUA, Greece \\ 15780 Zografos, Athens, Greece \\ Tel: +3017723570 \\ Fax:+3017723571 \\ e-mail:itat@central.ntua.gr
}

\begin{abstract}
This is an account of work done within the framework of CILC (Clothing Industry's Logistics and Communications) research project and the REALMS II (Reengineering Application Integrating Modelling and Simulation) Esprit project. The researchers' efforts have been focused on using the techniques and tools of business process simulation for the evaluation of business process re-engineering results. Two similar case studies are being demonstrated, one in the Garment Manufacturing Industry and another in the Metals Industry. The methodology followed consists of the following architectural blocks: Model development using a combination of IDEF0 diagrams and GRAI methodology, intermediate model depiction of the process to be simulated using IDEF3 diagrams, simulation model construction using the ARENA simulation software and SIMAN language, simulation run and evaluation based on a selected performance indicators set.
\end{abstract}

\section{Keywords}

Business Process Re-engineering, Extended Enterprise, Enterprise Modelling, Simulation.

\section{INTRODUCTION}

A new trend is the use of Business Process Simulation in the evaluation phase of re-engineering projects. Simulation is a tool that characterises a system, and provides means for evaluating potential results depending on changes of environmental variables(Schriber, 1987). Once the simulation of the solution has 
been conducted and the outcomes evaluated, decisions can be made. These decisions involve selecting the future course of action that will have the highest probability of achieving the desired result. Finally, the simulation model should be used to continually monitor and evaluate the process for continuous improvement.

Simulation is the only proven methodology that allows to incorporate all of the activities, resources, business rules, workload, assumptions, and other characteristics of a process into one model, and to test the impact of changes in assumptions or other elements on the behaviour and performance of the process (Helen's Club SA, NTUA, 1996). Virtually any performance criterion can be examined with simulation. Typical performance criteria are process cycle times, queue times, resource utilisation, activity costs or throughput (Shannon et al, 1981). Since simulation models mimic the actual process' response to events happening over time, it is possible to depict reality in a reliable way.

BPR practitioners have not been widely using proven quantitative analytical techniques to analyse and design business processes. The result is that few BPR projects implement new process designs based on a consideration of reliable performance metrics or reliable expected differences between competing alternatives (Furley, Garlitz. Kelleher, 1993). The methodology presented in the following chapters tries to cover the shortcomings of older approaches by using the concept of discrete simulation in conjunction with enterprise modelling and performance evaluation.

\section{PROPOSED METHODOLOGY}

A proposed methodology on the evaluation of a selected business process using Simulation can be seen in Figure 1.

The first step is the modelling of the new system with the use of IDEF-0 diagrams. IDEF provides disciplined ways of describing the structure of a system or organisation. IDEF-0 is a language for describing activities or processes and how they relate. Since understanding hierarchy is important in understanding complex systems, IDEF-0 is particularly useful because it includes hierarchy as an element of its modelling capability. IDEF-0 supplies the structure that exists among processes and provides the framework for simulation.

Although IDEF-0 is a helpful tool as a first stage of the approach, it cannot take into account the temporal dimension of the system. IDEF-3 is ideal to perform such an analysis, using its process schematics. IDEF-3 process schematics are the primary means for capturing, managing, and displaying process-centred knowledge. These schematics provide a graphical medium that helps domain experts and the analysis group communicate knowledge about processes. This includes knowledge about events and activities, the objects that participate in those 
occurrences, and the constraining relations that govern the behaviour of an occurrence. IDEF-3 combines the benefit of compatibility with the IDEF-0 methodology with this of being an ideal intermediate state for transition to the simulation process model.

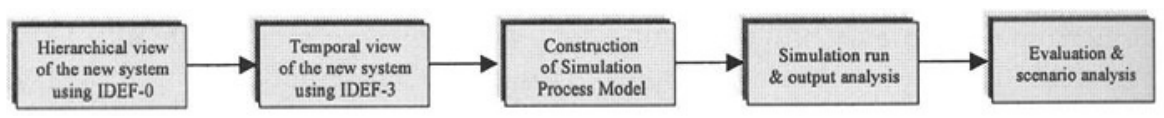

Figure 1 A Proposed Methodology.

The third stage refers to the construction of the process simulation model. In the proposed methodology, discrete event simulation is used. Discrete event simulations replicate processes as a sequence of events where each event has a beginning point and an ending point usually measured by time. Associated with these discrete points in time are state variables that measure the state of the process being simulated. Therefore, as a simulation proceeds through a series of events, the process under simulation will be viewed as a series of state changes. Analysis can focus either globally or locally as designed in any particular simulation. The software tool used, ARENA 2.2 for Windows, is a discrete event simulator.

After the construction of an accurate model which presents reality in a satisfactory way, the simulation run takes place. The simulation run is repeated for a number of times that can guarantee statistically acceptable results. In these results, the analysis of the output data is based.

The final step is the evaluation process with the use of pre-defined performance indicators which express the company's priorities and strategic orientation. The flexibility of the simulation approach permits the calculation of a variety of performance indicators related to time, cost or quality.

\section{CASE STUDIES}

\subsection{Greek Garment Manufacturing Company Study}

The company under discussion was founded in 1976 by a talented stylist. One of the main domains that the company has been interested in applying reengineering methods is the Domestic Customer Order Fulfilment Process (DCOFP), part of the Customer Order Flow process. The reasons that have pushed the decision makers to this direction are:

Serious process functioning problems that led to delays and long throughput times from the raw material acquisition to product-to-shelf point, weakness in successfully applying other time depreciation methods, continuously increasing process cost, need to achieve quick response to customer needs in order to gain a comparative advantage amongst other organisations trying to survive in a very 
competitive business environment., need for speeding up the Order Data Processing Procedure (ODPP) which will lead to an earlier production triggering, sales Person Route number reduction

The following IDEF-0 diagram presents the system part of which was analysed in the simulation study.

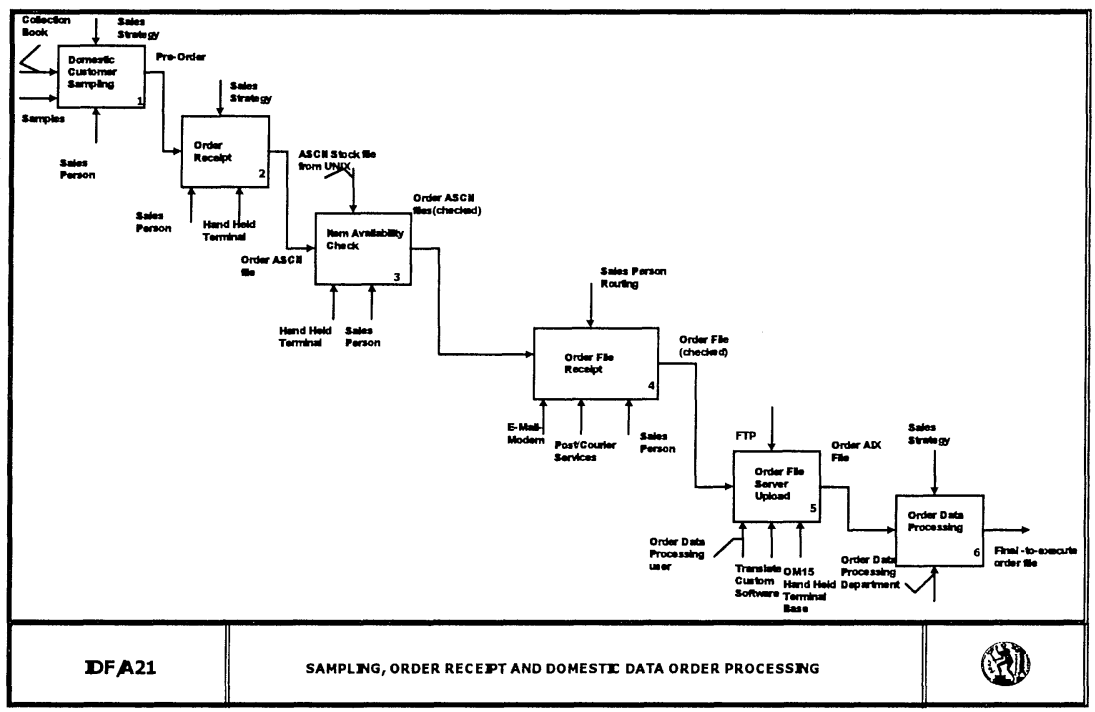

Figure 2 The IDEF-0 Model in the Garment Manufacturing Company.

After the construction of the related IDEF-0 diagram, and with the help of the corresponding IDEF-3 diagram, the simulation process model was designed.

\subsection{Greek Aluminum Products Manufacturer Study}

The company under discussion is an SME, considered to be one of the most successful in its sector at a European level, with a profit of about 10 billion drachmas.

One of the re-engineering areas in the company was selected to be the customer offer sub-process. In the situation before BPR the company was utilising fax and telex machines in order to receive the incoming messages. These messages were forwarded to the salesmen via simple mail after a day. Sometimes the messages were not sent to the appropriate people, a fact which caused delay for the customer response. The customer order request was performing an interdepartmental workflow and it finally returned to the corresponding sales-person. The next step was the preparation of the customer offer and finally it was forwarded to the secretaries for further communication with the customer. In the new situation, the installation of a fax-server, a mail-server and telex-server was decided as 
necessary, in order to handle electronically the incoming and outgoing messages. The activities of the new system which were analysed, and for which an IDEF-0 and IDEF-3 diagram was constructed, are the following:

A11 (To Precise the Request), A111 (Identification of Basic Technical Information), A12 (To Study The Technical Feasibility), A121 (To Check If the Specs Correspond to a Standard Product), A13 (To Define the Price and the Delivery Date), A131 (To Define a Production Date \& Calculate Cost), A132 (To Check the Acceptability of the Date), A133 (To Calculate a Delivery Date \& Price), A134 (To Negotiate Date \& Price With the Customer), A135 (To Negotiate Date Inside the Company).

The related IDEF-3 diagram shown in Figure 3 was designed based on the above activities and the corresponding IDEF-0 diagram. However, its most important advantage was the temporal dimension it presents which helped in the transition of the IDEF- 0 models to process simulation models of ARENA.
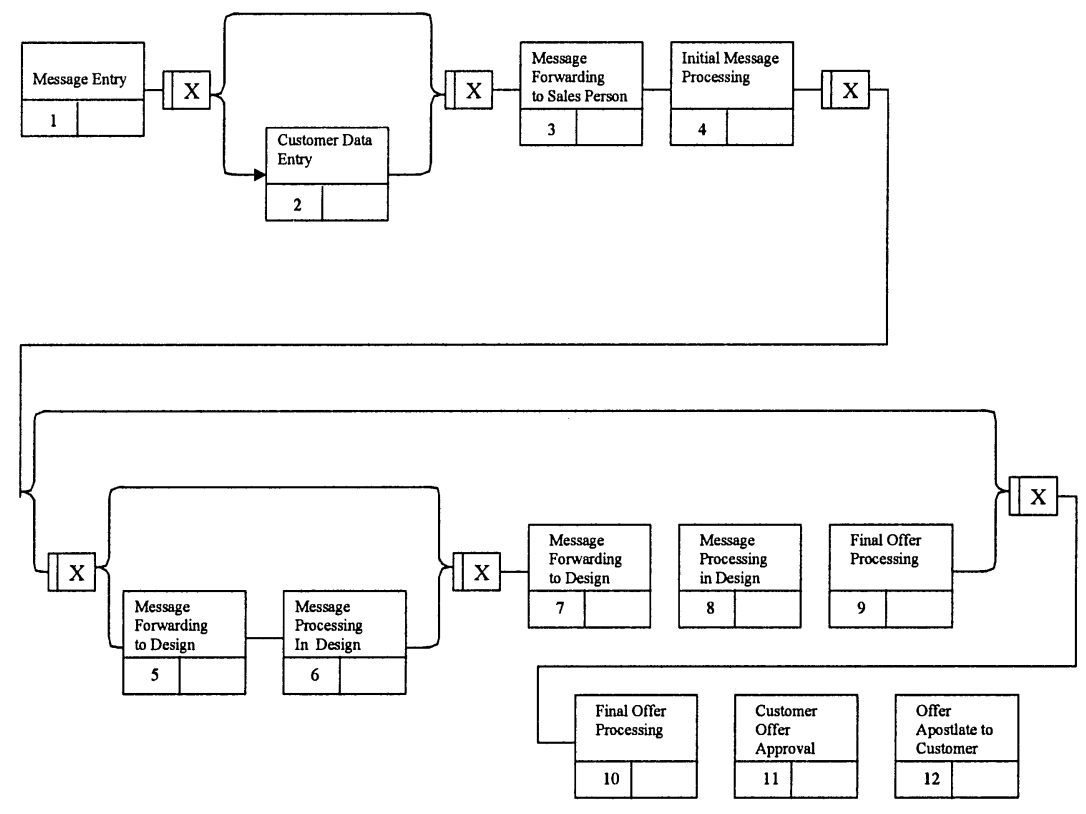

Figure 3 IDEF-3 Diagram.

Part of the final simulation model can be seen in Figure 4. 


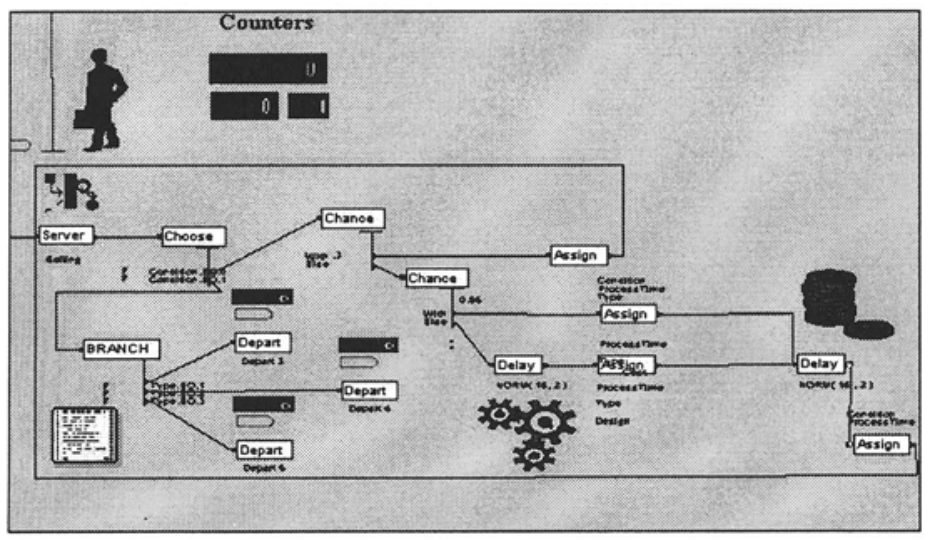

Figure 4 The Simulation Model in the Aluminum Company.

\subsection{Evaluation of Results}

The evaluation of results was based on a set of pre-defined performance indicators in the two companies. In both case studies, the most important, process level indicators that were selected are the following:

- Lead time of the process examined. This lead time affects the total lead time which is offered to the customer by the company

- Cost of creation of one customer offer or order. This is the translation of operational indicators into cost figures as well as a productivity indication.

- Number of entities in queues. The queues is a parameter that affects the reliability of the whole system which is being examined.

Comparison with queueing theory models was carried out in the case of the Aluminum company. A more careful analysis of the system under discussion proved that it could be approximated with small error by an $\mathrm{M} / \mathrm{G} / \mathrm{r}$ multi-server theory concerning the sales persons involved in the customer offer process. The Cosmetatos approximation formula shown below (Cosmetatos, 1976) was used for the validation of the simulation results.

$$
W\left(r_{j}, v\right) \simeq\left(\frac{1+v^{2}}{2}+\frac{\left(1-v^{2}\right)(1-\rho)\left(r_{j}-1\right)\left(\sqrt{ }\left(4+5 r_{j}\right)-2\right)}{16 \rho r_{j}}\right) W\left(r_{j}, 1\right)
$$

The calculation of the above formula generated results of very small relative percentage error compared with these achieved by the use of simulation (the average queue time was found to be 1.1565 hours, whereas the simulated time was 1.159 hours). These findings offered to the company an alternative of calculating the parameters of the particular system, in an easier way. However, the advantages 
of business simulation can be fully realised in the study of more complicated systems.

\section{CONCLUSIONS AND RESEARCH STATUS}

The use of business process simulation helped both organisations to evaluate the results of business process re-engineering, without having to wait for the stabilisation of the new system. In the case of the Aluminum company, the improvement in the time of the creation of one customer order was more $100 \%$ (from 12 hours to 4.5 hours in some cases of offers) and the cost reduction reached $75 \%$. Simulation runs were decided to be carried on for future alterations of the process, monitoring and fine-tuning.

Both of the projects on which the case studies were based have come to an end. Appropriate systems of data monitoring have been introduced in order to help the companies participate in a continuous improvement programme.

\section{REFERENCES}

1. Cosmetatos, G (1976) Some Approximate Equilibrium Results for the MultiServer Queue (M/G/r). Operational Research Quarterly, Volume 27, No 3, I, 615-620.

2. Furley, T.R. and Garlitz, J.L. and Kelleher, M.L. (1993) Applying Information Technology to Reengineering, Planning Review, 11, 22.

3. Helen's Club SA, NTUA (1996) Exrtended Network Enterprises in the Clothing Industry. Research funded by the Greek Ministry for Development.

4. Schriber, T.J. (1987) Applying Software Engineering to Simulation. Simulation, Volume 10., No1, 13-19.

5. Shannon, R. E, Long, S. S. and Buckles, B. P. (1981) Operation Research Methodologies in Industrial Engineering. AIIE Transactions, Volume 12, No 4, 364-367.

\section{BIOGRAPHY}

I.P. Tatsiopoulos is an Assoc. Professor in Production Planning and Control and Manufacturing Information Systems. His academic interests are computer-aided production management, logistics and the design of production systems. 